\title{
hercoe e e
}

REVISTA DE DIDÁCTICA ESPAÑOL COMO LENGUA EXTRANJERA

Este artículo fue publicado en Foro hispánico: revista hispánica de Flandes y Holanda (1994), núm. 6., páginas 65-75 (ISSN: 0925-8620), en una entrega dedicada a "Aproximaciones a cuestiones de adquisición y aprendizaje del español como lengua extranjera o lengua segunda", coordinada por Peter Slagter.

marcoELE reedita en versión en electrónica con las correspondientes autorizaciones y agradece al profesor Slagter y a los autores de este trabajo las facilidades brindadas.

1.

El propósito fundamental de este articulo es explicar por qué pensamos que la reflexión consciente (consciousness raising) sobre las

reglas gramaticales de la lengua objeto puede favorecer la adquisición

de ésta. Por un lado, contamos para sustentar esta opinión con evidencias empíricas que apuntan a que este tipo de práctica pedagógica favorece enormemente el dominio de la L2 (Castañeda, tesis en elaboración) y por otro, los modelos de adquisición que proponen la mayora de los investigadores no Krashen, desde luego tienen en cuenta la eficacia de la reflexión consciente $(\mathrm{RC})$.

Nosotros también propondremos un modelo menos ambicioso, que pretende explicar los efectos de la utilización en el aula de la reflexión consciente, tanto deductiva como inductivamente (en el modelo somos deudores por supuesto de E. Bialystok (1978), M. Sharwood Smith $(1988,1991)$ entre otros y sobre todo de Rod Ellis (1992), pero no adelantemos acontecimientos.

En definitiva, vamos a tratar de exponer:

- En qué consiste eso que los teóricos denominan reflexión consciente sobre aspectos formales de la lengua objeto.

- Cómo se incluye este instrumento pedagógico en un modelo general de adquisición.

\section{- Cómo y cuándo lo usamos y}

- Qué efectos pretendemos que tenga en la conducta del aprendiz.

Empezamos pues con la definición de consciousness raising que, en palabras de M. Sharwood Smith (1988) son "medios por los cuales hacemos conscientes a los aprendices de ciertos aspectos de la gramática. " Esta definición puede completarse con la opinión de Ellis de que el propósito que nos lleva a equipar al alumno con la comprensión de un rasgo gramatical específico es el de desarrollar 
en él un conocimiento declarativo explícito (un saber qué) más que un conocimiento procedural implícito (un saber cómo). El paso de uno a otro conocimiento ser objeto de posteriores reflexiones.

Debemos aclarar que nosotros vamos a utilizar el término RC de forma no totalmente coincidente con lo que Sharwood Smith (1988) o Bialystok (1978) proponen, sino que nuestra concepción de RC abarcara tanto los medios utilizados en clase para realzar ciertos aspectos gramaticales del input, como lo que los autores citados llaman introspección consciente, es decir, el trabajo mental que el aprendiz realiza a partir del input suministrado.

En efecto, se trata de utilizar en el aula ciertas estrategias de instrucción que centren la atención del aprendiz en regularidades específicas de la lengua, bien con el propósito de que, inductivamente, fabriquen reglas que puedan ser aplicadas a otros contextos en los que ese determinado aspecto gramatical aparezca o pueda aparecer (es decir, por ejemplo, le damos una serie de frases al alumno en las que hay diferencias entre "mi..." y "el mío..." y entre todos extraemos una posible regla de uso); o bien con el propósito de que, a partir de la presentación explícita de una regla, por ejemplo, "la diferencia entre " mi" / "el mío" es la siguiente: utilizo mío cuando ya sé de lo que estoy hablando y no necesito repetir", ellos la comprueben con ejemplos y la utilicen en actividades que necesiten de esa distinción para ser resueltas. Nosotros nos centraremos en el primer tipo de uso de RC, el uso inductivo, aunque pensamos que ambos son igualmente válidos; (un problema distinto ser saber qué contenidos se adaptan mejor a un tipo y qué contenidos a otro.)

Si partimos de la asunción de que todo conocimiento lingüístico está estructurado (del modo que sea), parece lógico deducir que cuanto mejor se conozca la estructura de ese conocimiento, mayor posibilidad de aplicación a distintas situaciones tendrá; mientras que si se posee la misma información lingüística de un modo no analizado, la posibilidad de aplicación a contextos nuevos ser bastante limitada, pues se ha aprendido holísticamente como una muestra, un "trozo" de lengua del que se ignora el alcance en otras situaciones. (Si yo me aprendo sin analizar "Me gusta la paella y la sangría", sólo podré utilizar esta frase en muy determinadas situaciones, si la analizo podré usar "me gusta" en muchos otros casos, por ejemplo.)

Queremos señalar que siempre hay algún tipo de reflexión consciente en la adquisición de la L2; dice textualmente Richard W. Schmidt que un aprendizaje subliminal de la lengua es imposible. Así, para llegar a poseer cierta competencia gramatical en la lengua objeto, el alumno formula hipótesis a partir de los datos a los que se encuentra expuesto; es decir, construye hipotesis que después generaliza, compara nuevos datos con los que ya posee para confirmarlos o negarlos, amplía o reduce el ámbito de esa regla transitoria que se había formulado, etc. Esto es especialmente cierto en el caso de los aprendices adultos, ya que, como señala entre otros Bley-Vroman (1986), el aprendizaje de una lengua por un adulto no se diferencia esencialmente de otros aprendizajes; 
de hecho, se utilizan mecanismos cognitivos similares a los que se aplican a sistemas generales de resolución de problemas.

En definitiva, un adulto no puede evitar comportarse del modo en que se comporta en el aprendizaje de la lengua, esto es, utilizando la reflexión consciente.

Si los aprendices van a reflexionar con nuestra ayuda o sin ella, no parece incoherente intentar acortar y facilitarles este proceso de descubrimiento de reglas, proporcionándoles los datos adecuados, en el momento adecuado, así como los medios para poder manejarlos e interpretarlos. Es decir les ayudamos y les enseñamos a pensar.

2.

Si en nuestra definición de RC hemos incluido, además de la introspección consciente, "los medios a través de los cuales podemos hacer a los aprendices conscientes de la gramática", parece sensato que intentemos definir cuanto antes el término "gramática" porque, como han dejado claro M. José Hernández y Lourdes Díaz (1993) por un lado y Ventura Salazar (1992) por otro, hacemos referencia a cosas muy distintas cuando decimos:

- La gramática del español es muy difícil

- La gramática para extranjeros de Atilano Sancho no explica bien el subjuntivo

O:

\section{- Erika tiene muchas dudas de gramática}

En el primer caso estamos hablando del funcionamiento interno de una lengua, es decir, de la competencia de que dispone un hablante nativo. En el segundo caso nos referimos a una descripción gramatical precisa, a un compendio de reglas y principios, elaborados desde una perspectiva teórica concreta, que pretende explicar el funcionamiento de una lengua a aprendices extranjeros. Y, con la tercera acepción de la palabra gramática, señalamos el conocimiento más o menos interiorizado que dispone un aprendiz de esas reglas y principios lingüísticos. A partir de ahora denominaremos $\mathrm{G} 1$ a la gramática objeto, es decir, el funcionamiento interno y no escrito de una lengua, G2 a la gramática pedagógica y G3 a la gramática de la interlengua del aprendiz, es decir, a la representación mental que éste posee de la G1.

Partiremos de una presuposición teórica que, justificada o no, subyace a toda investigación sobre el aprendizaje o la enseñanza de lenguas: el lenguaje es un comportamiento gobernado por reglas y la comunicación humana es posible gracias a una red de sistemas que pueden ser descritos en términos de reglas lingüísticas (Sharwood Smith, Rutherford, 1988). 
Cada modelo teórico formula y analiza estas reglas de una forma diferente y así, el marco gramatical al que los aprendices deben hacer frente varía en función del modelo teórico que haya escogido el autor del manual o la gramática pedagógica. Desde nuestro punto de vista el análisis gramatical más convincente es aquel que da cuenta de los fenómenos lingüisticos desde su dimensión pragmático-discursiva y, por lo tanto, partimos de la concepción de la "regla" que subyace en manuales como Intercambio o en la Gramática comunicativa del español de Francisco Matte Bon, donde las reglas son consideradas más allá de una mera dimensión sintáctica.

Pero no nos alejemos del tema: decíamos que el lenguaje es un comportamiento gobernado por reglas y que éstas se pueden hacer explícitas sobre el papel con distintos objetivos, unos de los cuales es facilitar a los estudiantes el aprendizaje de una L2. Siguiendo a Rutherford \& Sharwood Smith, podemos afirmar que en la gramática de la interlengua del aprendiz (G3) se va creando un sistema de reglas psicológicas (RP) que son el correlato mental de las reglas lingüisticas (RL) deliberadamente aprendidas a partir de un proceso analítico de reflexión consciente. Este sistema es lo que los teóricos han Ilamado gramática mental, gramática psicológica o gramática de la interlengua (fig. 1).

G1 (Gramática objeto)

G2 (Gramática pedagógica)

G3 (Gramática de la interlengua)

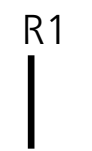

RL1

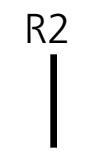

RL2

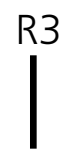

RL3

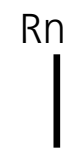

$\mathrm{RLn}$

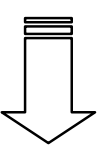

$\mathrm{RPn}$

R: Regla

RL: Regla Lingüistica

RP: Regla Psicológica

Por supuesto que este modelo en el que cada RL tiene su correlato en la gramática de la interlengua del aprendiz en una RP, pertenece, insistimos, a un modelo de enseñanza explícita de la gramática: al alumno se le hace consciente de la reglas explícitamente, esto es, contando en todo momento con su reflexión e introspección para incorporar dichas reglas a su sistema mental de la lengua objeto. Desde ahora, pues, podemos decir que nuestra propuesta se engloba en lo que Rutherford y Sharwood Smith Ilaman Hipótesis de la Gramática Pedagógica y que puede formularse más o menos así:

"Las estrategias de instrucción que llevan la atención del aprendiz hacia las regularidades estructurales del lenguaje pueden, bajo ciertas circunstancias, aumentar el nivel de adquisición en mayor grado que en circunstancias de acercamiento natural a la lengua donde la atención en la forma es mínima y esporádica. " 
Pero, cómo se produce ese paso que va desde la G2 a la G3? O, dicho de otro modo, ¿qué mecanismos mentales permiten crear una RP a partir de datos 0 , tal vez, de una RL? Para empezar diremos que los modelos teóricos no pretenden reproducir exactamente los mecanismos que tienen lugar en la mente del aprendiz, sino solamente dar una explicación que nos pueda ayudar a comprenderlos y a diseñar currículums, clases y actividades que de alguna forma puedan tenerlos en cuenta para facilitar el aprendizaje. Los modelos explicativos son muchos y, en general, todos pretenden aclarar, con un mayor o menor grado de escepticismo, qué fases, estrategias y procesos tienen lugar desde la exposición a la lengua objeto hasta la producción fluida y espontánea de esta L2.

Nosotros nos centraremos en mecanismos que intentan explicar el espacio que hay entre las RL y la posible incorporación de éstas a través de ejemplos a la gramática mental o conocimiento explícito del aprendiz; y, secundariamente, nos referiremos a cómo este paso influye indirectamente en la posterior automatización de las reglas.

Como se puede ver, en el primer esquema que presentamos, ese espacio está marcado con líneas discontinuas y no por flechas que indiquen una dirección única, y lo hemos hecho así por dos motivos: en primer lugar, porque la representación mental en la G3 del alumno quizá no tenga que ver directamente con las RL y sí con la interpretación que el aprendiz ha hecho de los datos a los que ha sido expuesto; (de hecho, no sabemos exactamente en qué consiste esa representación mental). $Y$, en segundo lugar, porque hay, en realidad, dos maneras de realizar ese camino: de forma deductiva y de forma inductiva.

1. En la enseñanza explícita deductiva, se expone la regla lingüística en primer lugar y después se ofrecen ejemplos que permiten al aprendiz contrastar la regla con los datos.

2. En la enseñanza explícita inductiva, por el contrario, se ofrecen al alumno los datos lingüísticos para que pueda, a partir de ellos, construir una hipótesis provisional de la RL que ser incorporada a su interlengua. "El estudiante, entonces, proyectará deductivamente esa regla en las nuevas muestras de lengua pertinentes que, si la contradicen, darán lugar a otra regla que la sustituya y a un consiguiente progreso de la interlengua." (Ventura Salazar, 1992). Es decir, en el primer caso el proceso es unidireccional, de la RL a la RP y, en el segundo, el camino es de ida y vuelta, coexisten momentos de inducción y de deducción pero es la inducción la que dispara el proceso.

Aunque ambas posturas nos parecen perfectamente válidas, es sin embargo la primera (enseñanza deductiva) la que tradicionalmente se ha impuesto, y se ha desdeñado la segunda probablemente porque la combinación de lo explícito y lo inductivo ha sido considerada durante mucho tiempo como imposible en la enseñanza de lenguas. Esta última perspectiva, estamos convencidos de ello, es sin embargo mucho más satisfactoria para ayudar al aprendiz a construir muchas 
reglas de la gramática de su interlengua. En efecto, parece bastante plausible suponer, como hace Sharwood Smith (1991) que los aprendices no interiorizan las RL, sino que lo único que interiorizan son "datos crudos", ejemplos de la regla, que deben ser procesados y convertidos en representaciones mentales (RP). En este sentido, cuando decimos que una RP es el "correlato mental" de una RL, debemos suponer que tal correlación no significa que la representación mental del alumno corresponda exactamente a la formulación de la RL, sino que éste es capaz de interpretar adecuadamente datos lingüisticos explicables en términos de una determinada RL. Evidentemente, una presentación explícita inductiva de contenidos gramaticales se adecua mucho más que la deductiva a esta realidad.

Para intentar aclarar mejor los procesos que ocupan el espacio entre la G2 y la G3 hemos elaborado el segundo esquema. Procuraremos mostrar cuáles son los procesos que se llevan a cabo cuando la regla es presentada directamente y cuando ésta se presenta de forma inductiva, y veremos qué papel desempeña la reflexión consciente en ambos casos.

\section{ESQUEMA 2}

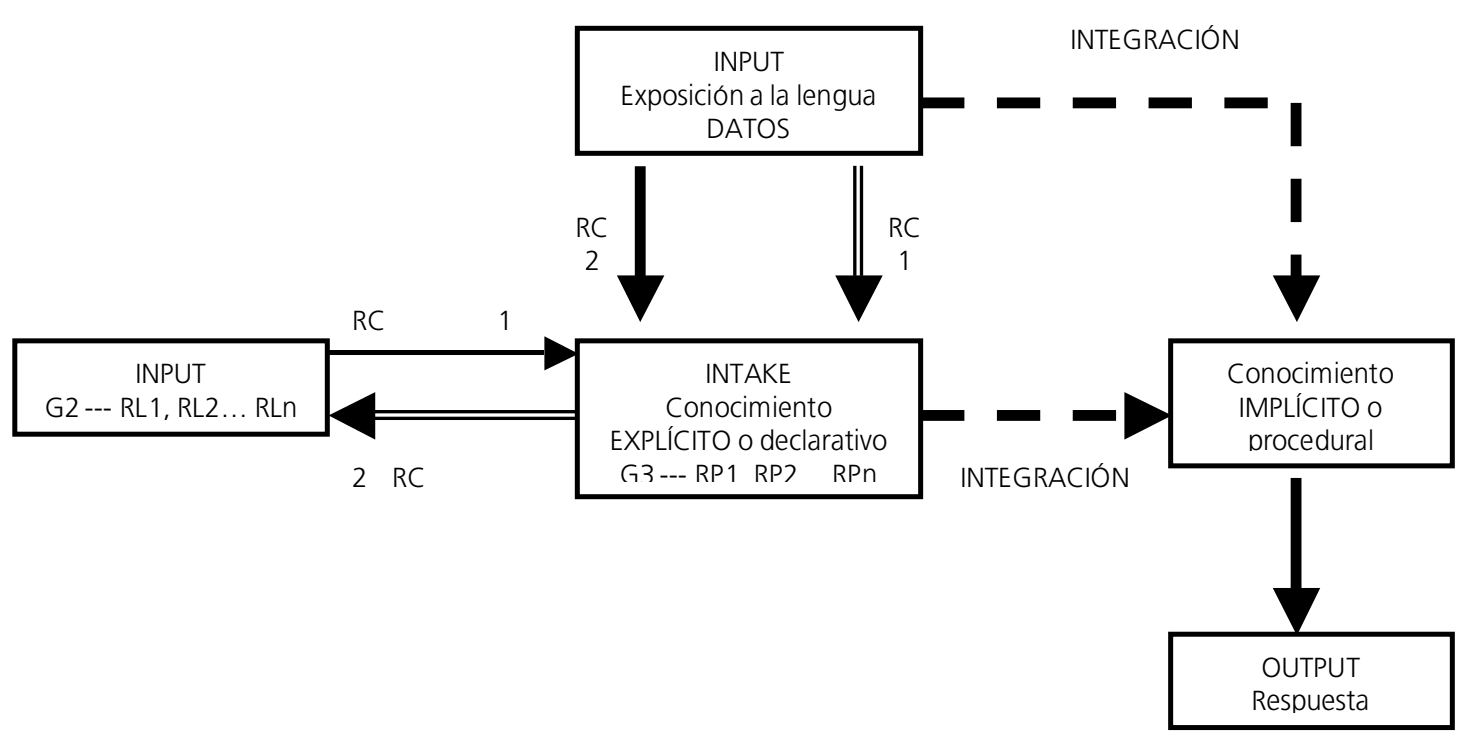

PROCESO DEDUCTIVO:

PROCESO INDUCTIVO:

RC= REFLEXIÓN CONSCIENTE

Proponemos que los procesos que se llevan a cabo en la práctica inductiva pueden ser los siguientes:

Partimos del Input, datos que, en algún grado, han sido manipulados, de modo que la ocurrencia del aspecto gramatical que nos interesa sea muy alta. Es decir, "realzamos" el input mediante la frecuencia de aparición y/u otros 
procedimientos menos elaborados como el empleo de la "negrita" para señalar los ítems a los que queremos que presten atención.

El alumno se "da cuenta" y "compara", i. e. utiliza la reflexión consciente para trabajar con esos datos (intake) en su G3. Como resultado de esa introspección consciente, el aprendiz elabora una hipótesis de regla lingüística que explique los datos evidentemente, para ello necesitar un metalenguaje, sea el que sea. Hay que decir que este paso no es absolutamente obligatorio; el aprendiz puede poseer la regla o la representación mental de ella y no ser capaz de verbalizarla. De hecho, puesto que, como ya hemos visto, lo que se interioriza son datos, ejemplos de regla, es probable que en su comportamiento "en la calle" el aprendiz se comporte del mismo modo (análisis de datos, elaboración de hipótesis...) sin haberse planteado siquiera la formulación de una posible regla aunque la posea.

Digamos pues, que la aplicación de la práctica inductiva es, en cierto modo, "la más natural ". Es decir, se adecua más a los procesos que de hecho ocurren en la mente del aprendiz.

La práctica deductiva, por el contrario, parte de las reglas lingüísticas y, posteriormente, de datos que son ejemplos de esas reglas, pero ya que son éstos últimos los que se interiorizan, la regla es algo secundario que puede servir de ayuda una vez que se han trabajado los ejemplos. Puesto que este procedimiento parte de algo secundario, i. e. la RL, parece, en principio, que este procedimiento es "menos natural".

Asimismo, la cantidad de reflexión consciente que exige el proceso inductivo es mayor, ya que son ellos mismos los que elaboran la regla (RL y RP) a partir de los datos, y suponemos que como consecuencia de ello la integración en el conocimiento explícito y posteriormente en el implícito queda así facilitada.

La RC, como hemos visto, se dirige esencialmente al desarrollo del conocimiento explícito (el que contiene las reglas analizadas); pero, a la hora de comunicarse espontáneamente en una situación concreta, el conocimiento que se pone en marcha es el conocimiento implícito (subconsciente, automatizado). Por tanto, la RC sólo tiene justificación si contribuye al desarrollo del conocimiento implícito, es decir, si contribuye a fomentar la habilidad del aprendiz para comunicarse.

Pues bien, aunque la RC no contribuye directamente a la adquisición de conocimiento implícito, sí lo hace de un modo indirecto, esto es, facilita la adquisición del conocimiento gramatical necesario para la comunicación. En efecto, la adquisición de conocimiento implócito se lleva a cabo mediante tres procesos que ya hemos visto:

- Darse cuenta (Noticing)

- Comparar (Comparing) 
En los dos primeros interviene la RC y el tercero tiene lugar en un nivel profundo de la consciencia del aprendiz y éste no es consciente de ello. Luego, de qué modo contribuye la RC a la adquisición de conocimiento implícito?

Rod Ellis propone dos modos fundamentales:

1. La RC contribuye a la realización de los procesos de "darse cuenta" y de "comparar", aunque no conduzca directamente a la interiorización.

2. El resultado de la utilización de la $\mathrm{RC}$ es conocimiento explícito, e incluso si el aprendiz no puede interiorizar el nuevo rasgo en el conocimiento implícito, la representación explícita que ha fabricado le puede ayudar a "darse cuenta" del rasgo en nuevos input, y de este modo conseguir finalmente su adquisición.

En definitiva, aunque la RC no tiene un resultado inmediato en la adquisición, sí posee un efecto retardado; y si de lo que se trata es de que el alumno tenga acceso a un determinado conocimiento gramatical sin necesidad de controlar conscientemente sus producciones, es decir, de que produzca espontáneamente en una determinada situación de comunicación, todas las evidencias sugieren que la percepción metalingüistica de regularidades estructurales de la lengua objeto afecta positivamente al desarrollo del conocimiento implícito.

A continuación incluimos un ejemplo de actividad que pretende mostrar cómo, a partir de la presentación de datos, se puede proponer a los alumnos reflexionar sobre cuál puede ser la regla que explique la oposición imperfecto / indefinido en esos casos. Evidentemente, para llevar a cabo un proceso de reflexión consciente y una posterior hipotesis de regla lingüística, es necesario algún tipo de metalenguaje con el que podamos organizar nuestras reflexiones. No hay, por el momento, ninguna evidencia empírica que apoye la necesidad de fomentar un conocimiento metalingüistico en nuestros alumnos, pero sí tenemos otros tipos de evidencia basados en la práctica cotidiana en el aula: cualquier tipo de explicación gramatical, deductiva, inductiva o mezcla de ambas, requiere de una terminología más o menos compleja con la que podamos hacer referencia a los hechos lingüísticos que pretendemos exponer. Y el tipo de terminología empleada depende en gran medida de la base teórica en la que se sustenta la explicación del libro de texto o la del profesor, de la misma manera que puede variar la explicación concreta de determinado fenómeno lingüístico; en este sentido, la controvertida oposición imperfecto / indefinido es sin duda un buen ejemplo.

Por otro lado, si, como acabamos de decir, la introspección consciente requiere un metalenguaje (más o menos explícito) que ayude a llevar a cabo la reflexión sobre la lengua, no parece excesivamente inútil dotar a nuestros aprendices con recursos terminológicos que les ayuden a organizar y reorganizar su G3 o gramática mental. 
En la actividad que presentamos, la solución al problema que se le plantea a los alumnos, (deducir una regla a partir de un texto que han debido crear antes) pasa por una reflexión que va más allá de lo meramente sintáctico y el metalenguaje necesario para verbalizarla puede ser negociado con los alumnos a partir de sus primeras hipótesis. Pero vayamos por partes. La tarea tiene tres pasos: en primer lugar, y después de haber leído el texto, deben escribir las formas del imperfecto de algunos verbos en un conjunto de frases que, aparentemente, no tienen conexión entre sí. Después tienen que decidir en qué lugar del texto original puede encajar cada una de esas frases; de esta manera ellos mismos van creando el texto completo desde lo que en principio no era más que una sucesión de acciones. Y por último, les pedimos que, a partir del texto original, las frases que han debido incorporar al relato y el resultado final, intenten explicar cuándo se utiliza cada una de las dos formas del pasado. Decíamos que el metalenguaje empleado para formular entre todos una regla puede ser negociado: no es necesario que el profesor imponga al final unos términos precisos, y su tarea puede limitarse a rechazar hipótesis incorrectas y a ordenar las propuestas acertadas de los alumnos. Para describir la función del indefinido aparecerán formulas como: "lo más importante", "las acciones principales", "lo que más interesa", etc. Y al imperfecto se le otorgarán funciones como "lo menos interesante", "descripciones", "explicar por qué", "las cosas menos importantes", etc. Todas estas propuestas son francamente útiles para dar cuenta de un hecho lingüístico que sólo puede ser explicado desde una visión pragmático-discursiva del lenguaje. Y son útiles, sobre todo, para ayudar a los aprendices a reorganizar su sistema mental de forma que haya espacio para un nuevo elemento.

Ésta es la historia de lo que la señora Horcajo hizo el martes pasado. Leela atentamente y pregunta el significado de las palabras que no entiendas. Fíjate en la forma verbal que se utiliza.

La señora Horcajo se levantó a las 8 (a). Fue a la cocina (b) y (c) puso la cafetera. Limpió un poco el fregadero y llamó después a su marido. (d). Tomaron juntos el café como cada día, y después ella lo despidió, como cada día, con un beso en la mejilla. (e) Llam después a una amiga y quedaron para ir juntas de compras. Salió de casa a las 10. (f) Estuvo con su amiga, (g), viendo algunas tiendas y fueron después a tomar un vino. Estuvieron hablando y a las 2 la señora Horcajo pensó: "ya es un poco tarde". (h). Miró otra vez el reloj, se sonrió y pidió otro vino (i). Por primera vez en su vida se tomó un vino a gusto.

- Aquel martes no (QUERER) hacer las aburridas tareas del hogar, así que

- porque su marido se (LEVANTARSE) prepararle el desayuno a las 8.15 y ella (TENER) que a casa a las 2.30 y ella, como cada da, (TENER) que prepararle la comida. 
- (SER) una mañana estupenda: (HACER) calor y no (HABER) mucho tráfico.

- que (ESTAR) bastante sucia

- porque no le (APETECER) volver y hacer la comida. (ESTAR) aburrida de la rutina diaria y también un poco cansada de su marido.

- como al señor Horcajo le gustaba el café recién hecho

- una mujer divorciada y muy simpática que se (LLAMAR) Sandra

- Siempre (SER) difícil despertarlo porque (DORMIR) muy profundamente.

\section{BIBLIOGRAFÍA}

Bialystok, Ellen, y M. Sharwood Smith (1985): "Interlanguage is not a state of mind: an evaluation of the construct for secondlanguage acquisition". Applied Linguistics, Vol. 6, Number 2.

Bialystok, Ellen (1991): "Un modelo teórico de aprendizaje de lenguas segundas". En: Muñoz Liceras, Juana (ed.).

Bley-Vroman, R. (1988): "The fundamental character of foreign language learning". En: Rutherford, W., and M. Sharwood Smith (eds.).

Castañeda Castro, Alejandro: Tesis en elaboración, Universidad de Granada.

Ellis, Rod (1992): Second Language Acquisition and Language Pedagogy. Clevedon: Multilingual Matters.

Green, P. and H. Karlheinz (1992): "Implicit and Explicit Grammar: An Empirical Study". Applied Linguistics, Vol. 13, Number 2.

Muñoz Liceras, Juana (ed.) (1991): La adquisición de las lenguas extranjeras. Madrid: Visor.

Rutherford, W., y M. Sharwood Smith (eds.) (1988): Grammar and second language teaching: a book of readings. New York: Newbury House / Harper and Row.

Salazar, Ventura: Gramática y enseñanza comunicativa del español / lengua extranjera. (En prensa).

Schmidt, R.W. (1990): "The role of consciousness in second language learning". Applied Linguistics, Vol. 11, Number 2. 
Sharwood Smith, M. (1993): "Input Enhancement in Instructed SLA". Studies in Second Language Research, 15, 165-179. 